S. Niwa

Nagoya Math. J.

Vol. 56 (1974), 147-161

\title{
MODULAR FORMS OF HALF INTEGRAL WEIGHT AND THE INTEGRAL OF CERTAIN THETA-FUNCTIONS
}

\author{
SHINJI NIWA
}

\section{§ 0. Introduction.}

Recently G. Shimura [1] constructed modular forms of integral weight from the forms of half integral weight. His construction is rather indirect. Indeed, he proved that the Dirichlet series, obtained from a form of half integral weight, multiplied by a certain $L$-function, corresponds to a modular form of an integral weight by means of the characterization of modular forms due to Weil.

In this paper, we shall give a more direct method of constructing modular forms of integral weight, using Siegel-Weil's indefinite theta series, and at the same time prove the conjecture related to the level of such forms (the former part of (A) in $\S 4$ of [1]). The relation of the theory of the theta series and Shimura's result was first pointed out by T. Shintani [2]. Indeed, he constructed, in contrast to Shimura's result modular forms of half integral weight, using an indefinite theta series, and showed that this correspondence is almost reciprocal to Shimura's. We note that our use of the theta series is, however, different from Shintani's.

Let $N$ be a positive integer, $\chi$ a character modulo $4 N$ and $\chi_{1}=\chi\left(\frac{-1}{*}\right)^{\lambda}$

with a positive integer $\lambda$. We denote by $H$ the complex upper half plane, and by $x=\left(x_{1}, x_{2}, x_{3}\right)$ an element of the vector space $\boldsymbol{R}^{3}$. For $g \in S L(2, \boldsymbol{R})$ we define a function on $R^{3}$ by $f(x)=\left(x_{1}-i x_{2}-x_{3}\right)^{2} \exp \left((-2 \pi / N)\left(2 x_{1}^{2}+x_{2}^{2}\right.\right.$ $\left.+2 x_{3}^{2}\right)$ ). For $\kappa=2 \lambda+1, z=u+i v \in H$ and for the lattice $L^{\prime}=Z \oplus N Z$ $\oplus(N Z / 4)$ in $Q^{3}$, we define a theta series $\theta(z, g)$ by

$$
\theta(z, g)=\sum_{x \in L^{\prime}} \bar{\chi}_{1}\left(x_{1}\right) v^{(3-x) / 4}\left(\exp \left(2 \pi i(u / N)\left(x_{2}^{2}-4 x_{1} x_{3}\right)\right)\right) f\left(\sqrt{v} g^{-1} x\right)
$$

where $\sqrt{v} \in \boldsymbol{R}$ is viewed as a scalar of the vector space $\boldsymbol{R}^{3}$, and 
$g \in S L(2, R)$ operates on $\boldsymbol{R}^{3}$ through the symmetric tensor representation, that is, $g x=\left(x_{1}^{\prime}, x_{2}^{\prime}, x_{3}^{\prime}\right) \in R^{3}$ is determined by

$$
g\left(\begin{array}{cc}
x_{1} & x_{2} / 2 \\
x_{2} / 2 & x_{3}
\end{array}\right)^{t} g=\left(\begin{array}{cc}
x_{1}^{\prime} & x_{2}^{\prime} / 2 \\
x_{2}^{\prime} / 2 & x_{3}^{\prime}
\end{array}\right) .
$$

This series is naturally derived from Weil's representation, and therefore a transformation formula in $z$ is guaranteed. Now, we consider the Petersson inner product of $\theta(z, g)$ with a cusp form of half integral weight with respect to $z$. Then, this inner product is a function of $g \in S L(2, R)$ which becomes a holomorphic modular form of integral weight on $H$. Such forms essentially coincide with those constructed by Shimura, as precisely stated below. Let $F(z)$ belongs to $S_{\star}\left(4 N, \bar{\chi}\left(\frac{N}{*}\right)\right)$; that is, $F(\sigma z)=\bar{\chi}(d)\left(\frac{N}{d}\right) j(\sigma, z)^{\star} F(z)$ for every $\sigma=\left(\begin{array}{ll}a & b \\ c & d\end{array}\right) \in \Gamma_{0}(4 N)$, where $j(\sigma, z)=\theta(\sigma z) / \theta(z)$ and $\theta(z)=\sum_{n=\infty}^{\infty} \exp \left(2 \pi i n^{2} z\right)$. Then, $G(z)=F(-1 / 4 N z)$ $\times(4 N)^{-\kappa / 4}(-i z)^{-\kappa / 2}$ belongs to $S_{k}(4 N, \chi)$, and has a Fourier expansion $G(z)=\sum_{k=1}^{\infty} a(k) \exp (2 \pi i k z)$. Determine $A_{1}(n)$ by the relation

$$
\sum_{n=1}^{\infty} A_{1}(n) n^{-s}=L\left(s-\lambda+1, \chi_{1}\right)\left(\sum_{k=1}^{\infty} a\left(k^{2}\right) k^{-s}\right),
$$

where $L(s, \chi)=\sum_{n=1}^{\infty} \chi(n) n^{-s}$. Then, putting $w^{\prime}=\xi^{\prime}+i \eta^{\prime}=-1 / 2 N w$ with $w=\xi+i \eta \in H$ and $\sigma_{w}=\left(\begin{array}{cc}\eta^{1 / 2} & \xi \eta^{-1 / 2} \\ 0 & \eta^{-1 / 2}\end{array}\right)$, we obtain

THEOREM. Asumme $\kappa \geqq 7$, then the function

$$
\Phi(w)=(2 N)^{\lambda}(-2 N w)^{-2 \lambda}\left(4 \eta^{\prime}\right)^{-\lambda} \int_{D} v^{k / 2} \bar{\theta}\left(z, \sigma_{4 w^{\prime}}\right) F(z) \frac{d u d v}{v^{2}} \quad(z=u+i v)
$$

of $w$ belongs to $\mathbb{S}_{x-1}\left(2 N, \chi^{2}\right)$ and has a Fourier expansion

$$
c \sum_{n=1}^{\infty} A_{1}(n) \exp (2 \pi i n w),
$$

where $c=(-1)^{\lambda} N^{\lambda / 2+1 / 4} 2^{-4 \lambda-\lambda / 2+3 / 2} \operatorname{Re}(\sqrt{2}-i)^{\lambda}$ and $\mathfrak{D}$ is the fundamental domain of $\Gamma_{0}(4 N)$ on $H$.

We denoted by $\mathbb{S}_{k-1}\left(2 N, \chi^{2}\right)$ the space of integral modular forms $\Phi$ of weight $\kappa-1$ satisfying $\Phi(\gamma w)=\chi^{2}(d)(c w+d)^{x-1} \Phi(x)$ for $\gamma=\left(\begin{array}{ll}a & b \\ c & d\end{array}\right)$ $\in \Gamma_{0}(2 N)$. Denote by $\widetilde{S}_{k-1}\left(2 N, \chi^{2}\right)$ the subspace of $\mathscr{S}_{k-1}\left(2 N, \chi^{2}\right)$ which 
consists of cusp forms. Then the function $\Phi$ in Theofem belongs to $\mathfrak{S}_{\kappa-1}\left(2 N, \chi^{2}\right)$ by virtue of the magnitude of the growth of $A_{1}(n)$.

This theorem assures that the above conjecture related to the level is true when $t=1$ with the notation of [1]. The more general cases, when $t$ is an arbitrary square-free positive integer, are obviously reduced to this case. Details will be given in $\S 3$.

We note that, in contrast to the situation in the theorem, the integral of $\theta$ with respect to $w^{\prime}$ is, as well known, an Einsenstein series according to the Siegel formula.

I wish to express my hearty thanks to Drs. T. Asai, T. Shintani and Y. Kitaoka, who gave me various important suggestions.

\section{\$1. Weil representation and theta series.}

The purpose of this section is to explain the transformation formula of $\theta$ introduced in the preceding section. For this purpose, we introduce Weil representation under the formulation by Shintani [2]. Let $Q^{(n)}$ be a nondegenerate $n$ by $n$ symmetric matrix of signature $(p, q)$ with rational coefficients, and let $\langle x, y\rangle={ }^{t} x Q y$ be the inner product of $x$ and $y$ in $\boldsymbol{R}^{n}$. For $\sigma=\left(\begin{array}{ll}a & b \\ c & d\end{array}\right)$ in $S L(2, \boldsymbol{R})$ and $f(x)$ in $L^{2}\left(\boldsymbol{R}^{n}\right)$, Weil representation $\sigma \rightarrow r_{0}(\sigma)$ is defined as follows :

$$
\begin{aligned}
& \left(r_{0}(\sigma) f\right)(x) \\
& =\left\{\begin{array}{l}
|a|^{n / 2} e[(a b / 2)\langle x, x\rangle] f(a x), \quad \text { for } c=0, \\
|\operatorname{det} Q|^{1 / 2}|c|^{-n / 2} \int_{R^{n}} e\left[\frac{a\langle x, x\rangle-2\langle x, y\rangle+d\langle y, y\rangle}{2 c}\right] f(y) d y,
\end{array} \quad \text { for } c \neq 0,\right.
\end{aligned}
$$

where $e[x]=\exp (2 \pi i x)$. Put $r(\sigma)=\varepsilon(\sigma)^{q-p} r_{0}(\sigma)$ with

$$
\varepsilon(\sigma)=\left\{\begin{array} { l } 
{ \sqrt { i } , } \\
{ i ^ { ( 1 - \operatorname { s g n } d ) / 2 } , } \\
{ \sqrt { i } ^ { - 1 } , }
\end{array} \quad \text { for } \left\{\begin{array}{l}
c>0, \\
c=0, \\
c<0 .
\end{array}\right.\right.
$$

Assume that $\langle x, x\rangle$ takes integral values on the lattice $L$ in $Q^{n}$. Denote the dual lattice of $L$ by $L^{*}$, and for $h \in L^{*} / L$ and $f \in S\left(R^{n}\right)$, define the series $\theta(f, h)$ by $\sum_{x \in L} f(h+x)$; then we obtain

Proposition 0 (Shintani). For $\sigma=\left(\begin{array}{ll}a & b \\ c & d\end{array}\right) \in S L(2, Z)$ such that 
$a b\langle x, x\rangle, c d\langle x, x\rangle \equiv 0 \bmod 2$ for all $x \in L$, we have

$$
\theta\left(r_{0}(\sigma) f, h\right)=\sum_{k \in L^{*} / L} c(h, k)_{\sigma} \theta(f, k)
$$

where $c(h, k)_{o}$ is equal to

$$
\delta_{k, a h} e[(a b / 2)\langle h, k\rangle]
$$

for $c=0$, and equal to

$$
\begin{aligned}
& |\operatorname{det} Q|^{-1 / 2} \operatorname{vol}(L)^{-1}|c|^{-n / 2} \\
& \quad \times \sum_{r \in L / c L} e[(1 / 2 c)(a\langle h+r, h+r\rangle-2\langle k, h+r\rangle+d\langle k, k\rangle)]
\end{aligned}
$$

for $c \neq 0$. Moreover, if $c \equiv c\langle x, x\rangle \equiv 0(\bmod 2)$ for every $x \in L^{*}$ and $c L^{*} \subset L$ are satisfied, then, for $d<0$ and $c \neq 0, c(h, k)_{\sigma}$ is given by

$$
\delta_{k, a h} \sqrt{i}^{-(p-q) \operatorname{sgn} c}|d|^{-n / 2} \sum_{r \in L / d L} e\left[\frac{b\langle h+r, h+r\rangle}{2 d}\right] .
$$

COROLLARY (Shintani) 0. Under the same assumption as above, assume that $f$ satisfies $r(k(\theta)) f=(\cos \theta-i \sin \theta)^{-x / 2} f$ for all $\theta$, where $k(\theta)$ $=\left(\begin{array}{rr}\cos \theta & \sin \theta \\ -\sin \theta & \cos \theta\end{array}\right)$ and $\kappa$ is a positive integer, and put $\theta(z, f, h)$ $=v^{-\kappa / 4}\left(r_{0}\left(\sigma_{z}\right) f, h\right)$; then we get the transformation formula

$$
(\sqrt{i})^{(p-q) \operatorname{sgn} c}(c z+d)^{-\kappa / 2} \theta(\sigma z, f, h)=\sum_{k \in L^{*} / L} c(h, k)_{o} \theta(z, f, k), \quad(c \neq 0) .
$$

Throuhout this paper we determine $\sqrt{z}=z^{1 / 2}$ by $-\pi / 2<\arg z^{1 / 2} \leqq \pi / 2$. Now, we give some examples and explanations for later use.

EXAMPLE 1. We consider the case $n=1, Q=(2 / N), L=N Z$ and $f(x)=\exp \left(-(2 \pi / N) x^{2}\right)$, then we have $p=1, q=0, L^{*}=Z / 2, r(k(\theta)) f$ $=(\cos \theta-i \sin \theta)^{-1 / 2} f$ and $\theta(z, f, 0)=\theta(N z)$, where $\theta(z)$ is defined in $\S .0$. From Corollary 0 , it follows for $\sigma=\left(\begin{array}{ll}a & b \\ c & d\end{array}\right) \in \Gamma_{0}(4 N)$ that $(\sqrt{i})^{\operatorname{sgn} c}(c z$ $+d)^{-1 / 2} \theta(N \sigma z)=c(0,0)_{\sigma} \theta(N z)$ and $c(0,0)_{\sigma}=(\sqrt{i})^{\operatorname{sgn} c} j(\sigma, z)(c z+d)^{-1 / 2}\left(\frac{N}{d}\right)$. We note that $c(h, k)_{\sigma}$ in Proposition 0 does not depend on $f$. We can interprete this representation by the so-called Fock representation. We define a map $I: L^{2}(\boldsymbol{R}) \rightarrow \boldsymbol{H}=L^{2}(\boldsymbol{C}, \exp (-\pi z \bar{z}) d z)$ by the integral transformation $(I f)(z)=\int_{R} k(x, z) f(x) d x$, where $f \in L^{2}(\boldsymbol{R})$ and $k(x, z)=\exp$ $\times\left(-\pi m x^{2}\right) e[x \sqrt{m z}] \exp \left((\pi / 2) z^{2}\right)$, then $I$ is bijective and maps the Hermit 
function $\left.\exp \left(\pi m x^{2}\right) \frac{d^{s}}{d x^{6}}\right|_{\sqrt{m} x} \exp \left(-2 \pi x^{2}\right)$ in $L^{2}(\boldsymbol{R})$ to the polynomial $z^{\varepsilon}$ in $H$ up to a constant multiple. Moreover one can easily check, by direct calculations, that $\operatorname{Ir}(k(\theta)) f=(\cos \theta-i \sin \theta)^{-1 / 2} M\left(e^{i \theta}\right) I f$, where $f \in L^{2}(\boldsymbol{R})$, $Q=(m)$ and $M\left(e^{i \theta}\right)$ is the map such that $M\left(e^{i \theta}\right) g(z)=g\left(e^{i \theta} z\right)$ for $g(z) \in \boldsymbol{H}$. In this way we can find a function $f_{1, \varepsilon}$ in $L^{2}(R)$ satisfying $r(k(\theta)) f_{1, \varepsilon}$ $=(\cos \theta-i \sin \theta)^{-(2 \varepsilon+1) / 2} f_{1, \varepsilon}$ for a positive integer $\varepsilon$. Namely, $f_{1, \varepsilon}(x)$ $=H_{c}\left(2 \sqrt{\pi m} x^{2}\right)$, where $H_{s}(x)=(-1)^{c} \exp \left(x^{2} / 2\right) \frac{d^{6}}{d x^{6}} \exp \left(-x^{2} / 2\right)$ is a socalled Hermite polynomial.

Put again $m=2 / N$ and let $L$ be as above; then $\theta\left(z, f_{1, \varepsilon}, 0\right)=\theta_{1, \varepsilon}(z)$ $=v^{-s / 2} \sum_{x=-\infty}^{\infty} H(2 \sqrt{2 N \pi v} x) \exp \left(2 \pi i N z x^{2}\right) \quad$ satisfies $\theta_{1, s}(\sigma z)=\left(\frac{N}{d}\right) j(\sigma, z)(c z$ $+d)^{s} \theta_{1,8}(z)$ according to the independence of $c(h, k)_{\sigma}$ to $f$. In the same way $\theta_{1, \varsigma}(-1 / 4 N z)=(2 N)^{\epsilon / 2} \times(\sqrt{-2 i z})^{2 \epsilon+1} \theta_{c}(z)$ can be shown with $\theta_{\epsilon}(z)$ $=(2 v)^{-\varepsilon / 2} \sum_{x=-\infty}^{\infty} \exp \left(2 \pi i x^{2} z\right) H_{\varepsilon}(2 \sqrt{2 \pi v} x)$.

Example 2. Next we consider the case $n=2$ and $Q=(2 / N)$ $\times\left(-2^{-2}\right)$, that is, $\langle x, y\rangle=(-4 / N)\left(x_{1} y_{2}+x_{2} y_{1}\right)$ and $L=4 N Z \oplus(N Z / 4)$, then $p=q=1, r=r_{0}$ and $L^{*}=Z \oplus(Z / 16)$, and $4 N L^{*}=L$ satisfies the assumption of proposition 0 . Put $L^{\prime}=Z \oplus(N Z / 4), h \in L^{\prime}$ : then for $\sigma=\left(\begin{array}{ll}a & b \\ c & d\end{array}\right) \in \Gamma_{0}(4 N), c(h, k)_{\sigma}=\delta_{k, a h}$ and $\theta\left(r_{0}(\sigma) f, h\right)=\theta(f, a h)$ are valid. If $f \in S\left(\boldsymbol{R}^{2}\right)$ satisfies $r(k(\theta)) f=e^{i \epsilon \theta} f$, and if we define $\theta_{2, \theta}(z, f)$ by

$$
\theta_{2, \varepsilon}(z, f)=\sum_{h \in L^{\prime} / L^{*}} \bar{\chi}_{1}\left(h_{1}\right) \theta(z, f, h)
$$

then we obtain

$$
\theta_{2, \varepsilon}(\sigma z, f)=\bar{\chi}_{1}(d)(c z+d)^{s} \theta_{2, s}(z, f)
$$

We explain how to find $f$ with this property. Put $Q=m \times(-2-2), m$ $>0$. We define partial Fourier transformation $F$ by

$$
\begin{gathered}
(F f)\left(x_{1}, x_{2}\right)=\sqrt{2 m} \int_{-\infty}^{\infty} f\left(x_{1}, t\right) \exp \left(4 \pi i m t x_{2}\right) d t \\
\left(F^{-1} f\right)\left(x_{1}, x_{2}\right)=\sqrt{2 m} \int_{-\infty}^{\infty} f\left(x_{1}, t\right) \exp \left(-4 \pi i m t x_{2}\right) d t
\end{gathered}
$$


One can easily check that $r(\sigma) f=F R(\sigma) F^{-1} f$, where $(R(\sigma) f)(x)=f\left(\left(x_{1}, x_{2}\right) \sigma\right)$, and so $r$ is a representation of $S L(2, R)$ although Weil representation is not always a multiplicative representation. Put $f^{\prime}\left(x_{1}, x_{2}\right)=\left(x_{1}+i x_{2}\right)^{\text {s }}$ $\times \exp \left(-2 m \pi\left(x_{1}^{2}+x_{2}^{2}\right)\right), f_{2, \mathrm{e}}(x)=\left(F f^{\prime}\right)(x)=\sqrt{2}(\sqrt{4 \pi m})^{-\cdot-1} H\left(\sqrt{4 \pi m}\left(x_{1}-x_{2}\right)\right)$ $\times \exp \left(-2 m \pi\left(x_{1}^{2}+x_{2}^{2}\right)\right)$; then $R(k(\theta)) f^{\prime}=e^{2 i \varepsilon \theta} f^{\prime}$ and $f_{2, \mathrm{~s}}$ has the required property. Generally, the Weil representation commutes with the action of the orthogonal group of $Q$ on $L^{2}\left(\boldsymbol{R}^{n}\right)$. In the present case, the elements of that group are diagonal matrices in $S L(2, R)$. Put $f_{\eta}\left(x_{1}, x_{2}\right)$ $=f_{2, \varepsilon}\left(\eta^{-1} x_{1}, \eta x_{2}\right)$ with $f_{2, \epsilon}$ above, and put $m=2 / N$. Put $\theta_{2, e}(z, \eta)$ $=\theta_{2, \varepsilon}\left(z, f_{\eta}\right)$. Then we have

$$
\begin{aligned}
\theta_{2, \varepsilon}(z, \eta)= & v^{(1-s) / 2} \sum_{x_{1}, x_{2} \in Z} \bar{\chi}_{1}\left(x_{1}\right) \\
& \times \exp \left(-2 \pi i u x_{1} x_{2}-\frac{N v}{4} \pi x_{2}^{2} \eta^{2}-\frac{4 v}{N} \pi x_{1}^{2} \eta^{-2}\right) \\
& \times H_{\bullet}\left(2 \sqrt{\frac{2}{N} \pi v}\left(x_{1} \eta^{-1}-\frac{N x_{2}}{4} \eta\right)\right) .
\end{aligned}
$$

Observing that $f_{2, \varepsilon}=F f^{\prime}$ and using the Poisson summation formula, we obtain a different expression of $\theta_{2, \varepsilon}$ :

$$
\begin{aligned}
\theta_{2, \mathrm{\varepsilon}}(z, \eta)= & \left(\sqrt{\frac{8 \pi}{N}}\right)^{\iota+1}(\sqrt{2 \pi})^{-1} i^{\bullet} \eta^{-\bullet-1} v^{-\varepsilon} \\
& \times \sum_{x_{1}, x_{2} \in Z} \bar{\chi}_{1}\left(x_{1}\right)\left(x_{1} \bar{z}+x_{2}\right)^{\bullet} \exp \left(-\frac{4 \pi}{N \eta^{2} v}\left|x_{1} z+x_{2}\right|^{2}\right) .
\end{aligned}
$$

EXAMPLE 3. We denote by $r_{0}^{(i)}$ the Weil representation in the vector space $V_{i},(i=1,2,3)$, and by $L_{i}, L_{i}^{*}, r^{(i)}, h_{i} \in L_{i}^{*}$ and $c_{i}\left(h_{i}, k_{i}\right)_{\sigma}$ corresponding lattices, etc. If $V_{3}$ is the orthogonal sum of $V_{1}$ and $V_{2}$, then $r_{0}^{(3)}$ $=r_{0}^{(1)} \otimes r_{0}^{(2)}, r^{(3)}=r^{(1)} \otimes r^{(2)}$, and $c_{3}\left(h_{3}, k_{3}\right)_{\sigma}=c_{1}\left(h_{1}, k_{1}\right)_{\sigma} c_{2}\left(h_{2}, k_{2}\right)_{\sigma}$ is obvious for $h_{3}=\left(h_{1}, h_{2}\right), k_{3}=\left(k_{1}, k_{2}\right)$. If $n=3, Q=\frac{2}{N}\left({ }_{-2} 1^{-2}\right)$ and $L=4 N Z$ $\oplus N Z \oplus(N Z / 4)$, then, according to preceding two examples, we have

$$
c(h, k)_{\sigma}=\delta_{k, a h}(\sqrt{i})^{\mathrm{sgn} c} j(\sigma, z)(c z+d)^{-1 / 2}\left(\frac{N}{d}\right)
$$

for $f \in L^{2}\left(\boldsymbol{R}^{3}\right), \sigma=\left(\begin{array}{ll}a & b \\ c & d\end{array}\right) \in \Gamma_{0}(4 N)$ and $h, k \in L^{\prime} / L$ with $L^{\prime}=\boldsymbol{Z} \oplus N \boldsymbol{Z}$ $\oplus(N Z / 4)$. Consequently, if $r(k(\theta)) f=(\cos \theta-\sin \theta)^{-x / 2} f$ is satisfied, then, defining $\theta_{\kappa}(z, f)=\sum_{h \in L^{\prime} / L} \bar{\chi}_{1}(h) \theta(z, f, h)$, where $\bar{\chi}_{1}(h)=\bar{\chi}_{1}\left(h_{1}\right), \quad h$ 
$=\left(h_{1}, h_{2}, h_{3}\right)$ and $\kappa=2 \lambda+1$ is an odd integer, we obtain

$$
\theta(\sigma z, f)=\bar{\chi}_{1}(d)\left(\frac{N}{d}\right) j(\sigma, z)(c z+d)^{\lambda} \theta_{\kappa}(z, f)
$$

by Corollary 0. One can take here $f_{1, \varepsilon}\left(x_{2}\right) f_{2, \lambda-s}\left(x_{1}, x_{3}\right),(\varepsilon=1, \cdots, \lambda)$, or their linear combinations for such $f(x)$. In view of $(x-i y)^{2}$ $=\sum_{\iota=0}^{\lambda}\left(\begin{array}{l}\lambda \\ \varepsilon\end{array}\right) H_{\lambda-\varepsilon}(x) H_{\bullet}(y)(-i)^{\natural}, f_{3}(x)=\left(x_{1}-i x_{2}-x_{3}\right)^{\lambda} \exp \left(-m \pi\left(2 x_{1}^{2}+x_{2}^{2}+2 x_{3}^{2}\right)\right)$ is available, too. On the other hand, the action of $S L(2, R)$ in $R^{3}$ defined in $\S .0$ gives an isomorphism of $S L(2, R)$ with the orthogonal group of $Q$. Let $g f \in L^{2}\left(R^{3}\right)$ be defined by $(g f)(x)=f\left(g^{-1} x\right)$ and $m=2 / N$; then $\theta(z, g)$ defined in $\S .0$ coincides with $\theta\left(z, g f_{3}\right)$. The action of $r_{0}(k(\theta))$ commutes with that of $g$ in $L^{2}\left(R^{3}\right), g f_{3}$ has the same property as $f_{3}$, and the required transformation formula of $\theta(z, g)$ is

$$
\theta(\sigma z, g)=\bar{\chi}(d)\left(\frac{N}{d}\right) j(\sigma, z)^{x} \theta(z, g) .
$$

Remark. We note that $f_{3}$ has the property $f_{3}(k(\theta) x)=e^{2 \lambda i \theta} f_{3}(x)$, and so $\theta(z, g k(\theta))=e^{-2 i \lambda \theta} \theta(z, g)$.

\section{§ 2. Construction of the modular forms identical with Shimura's (Proof of the theorem).}

Let $F(z)$ be in $S_{\star}\left(4 N, \bar{\chi}\left(\frac{N}{*}\right)\right)$. Since $F(z)$ is rapidly decreasing at each cusp of $\mathfrak{D}$, while $\theta(z, g)$ is at most slowly increasing there, the following integral is well defined:

$$
\Psi(w)=(4 \eta)^{-2} \int_{\varnothing} v^{\kappa / 2} \bar{\theta}\left(z, \sigma_{4 w}\right) F(z) \frac{d u d v}{v^{2}} .
$$

Put

$$
\Phi(w)=\Psi\left(-\frac{1}{2 N w}\right)(2 N)^{2}(-2 N w)^{-2 \lambda}
$$

(this is the function given in Theorem). Then, in view of

$$
\left(\begin{array}{ll}
a & b \\
c & d
\end{array}\right)\left(\begin{array}{ll}
x_{1} & x_{2} / 2 \\
x_{2} / 2 & x_{3}
\end{array}\right)\left(\begin{array}{ll}
a & c \\
b & d
\end{array}\right)=\left(\begin{array}{ll}
x_{1}^{\prime} & x_{2}^{\prime} / 2 \\
x_{2}^{\prime} / 2 & x_{3}^{\prime}
\end{array}\right)
$$

i.e. 


$$
\begin{aligned}
& x_{1}^{\prime}=a^{2} x_{1}+a b x_{2}+b^{2} x_{3} \\
& x_{2}^{\prime}=2 c a x_{1}+(a d+c b) x_{2}+2 b d x_{3} \\
& x_{3}^{\prime}=c^{2} x_{1}+c d x_{2}+d^{2} x_{3},
\end{aligned}
$$

and $a, d \in Z, c \in N Z / 2$ and $b \in 4 Z$ for $\tilde{\gamma}=\left(\begin{array}{ll}a & b \\ c & d\end{array}\right) \in \Gamma=\left(\begin{array}{ll}2 & \\ & 1 / 2\end{array}\right) \Gamma_{0}(2 N)\left(\begin{array}{cc}1 / 2 & \\ & 2\end{array}\right)$, both lattices $L=4 N \boldsymbol{Z} \oplus N \boldsymbol{Z} \oplus(N \boldsymbol{Z} / 4)$ and $L^{\prime}=\boldsymbol{Z} \oplus N \boldsymbol{Z} \oplus(N \boldsymbol{Z} / 4)$ are stable by $\tilde{\gamma}$, and $x_{1}^{\prime} \equiv a^{2} x_{1}(\bmod 4 N)$ for $x=\left(x_{1}, x_{2}, x_{3}\right) \in L^{\prime}$, so we obtain $\theta(z, \tilde{\gamma} g)=\chi^{2}(d) \theta(z, g)$ and consequently.

$$
\Psi(\gamma w)=\bar{\chi}^{2}\left(d^{\prime}\right)\left(c^{\prime} w+\mathrm{d}^{\prime}\right)^{2 \lambda} \Psi(w)
$$

for $\gamma=\left(\begin{array}{ll}a^{\prime} & b^{\prime} \\ c^{\prime} & d^{\prime}\end{array}\right) \in \Gamma_{0}(2 N)$, recalling the remark in the preceding section.

It It is easy to see that $\theta\left(z, \sigma_{4 w}\right)$ is slowly increasing on $H \times H$. Therefore, if $\Psi(w)$ is holomorphic on $H$, then we can conclude that $\Psi$ is an integral modular form of weight $2 \lambda$ and so is $\Phi$, too. The holomorphy of $\Psi$ is a direct consequence of Shintani's result

$$
\begin{aligned}
D_{g} \theta(z, g)= & \left\{4 v^{2}\left(\frac{\partial^{2}}{\partial u^{2}}+\frac{\partial}{\partial v^{2}}\right)-2 i \kappa v\left(\frac{\partial}{\partial u}+i \frac{\partial}{\partial v}\right)\right. \\
& \left.+\kappa\left(\frac{\kappa}{4}-1\right)+\frac{3}{4}\right\} \theta(z, g)
\end{aligned}
$$

where $D_{g}=\frac{1}{4}\left(\left(\begin{array}{rr}1 & 0 \\ 0 & -1\end{array}\right)^{2}+2\left(\begin{array}{ll}0 & 1 \\ 0 & 0\end{array}\right)\left(\begin{array}{ll}0 & 0 \\ 1 & 0\end{array}\right)+2\left(\begin{array}{ll}0 & 0 \\ 1 & 0\end{array}\right)\left(\begin{array}{ll}0 & 1 \\ 0 & 0\end{array}\right)\right)$ is the Casimir operator on $S L(2, R)$, (see [2], §1). By the Green's formula we have

$$
D_{g} \int_{D} v^{\kappa / 2} \bar{\theta}(z, g) F(z) \frac{\mathrm{d} u \mathrm{~d} v}{v^{2}}=\lambda(\lambda-1) \int_{D} v^{\alpha / 2} \bar{\theta}(z, g) F(z) \frac{\mathrm{d} u \mathrm{~d} v}{v^{2}},
$$

equivalently

$$
\left(\eta^{2}\left(\frac{\partial^{2}}{\partial \xi^{2}}+\frac{\partial^{2}}{\partial \eta^{2}}\right)-2 \lambda i \eta\left(\frac{\partial}{\partial \xi}\right)\right) \Psi(w)=0
$$

Put $\Psi(w)=\sum_{m=-\infty}^{\infty} a_{m}(\eta) \exp (2 \pi i m \xi)$. Then, from the above partial differential equation, we have $a_{m}(\eta)=b_{m} \exp (-2 \pi m \eta)+c_{m} \exp (-2 \pi m \eta)$ $\times \int_{0}^{\eta} \eta^{-2 \lambda} \exp (4 \pi m \eta) \mathrm{d} \eta,(m \neq 0)$ and $a_{0}(\eta)=b_{0}+c_{0} \eta^{-2 \lambda+1}$, where $\exp (-2 \pi m \eta)$ $\times \int_{0}^{\eta} \eta^{-2 \lambda} \exp (4 \pi m \eta) \mathrm{d} \eta=0\left(\eta^{-2 \lambda+1} e^{2 \pi m \eta}\right)$. On the other hand $\eta^{2} \Psi(w)=0(\eta+1 / \eta)$ uniformly in $\xi$ as will be showed. Thus $a_{m}(\eta)=0\left((\eta+1 / \eta) \eta^{-\lambda}\right)$, since 


$$
\int_{0}^{1} \eta^{2 \lambda}|\Psi(w)|^{2} \mathrm{~d} \xi=\sum_{m=-\infty}^{\infty}\left|a_{m}(\eta)\right|^{2} \eta^{2 \lambda}
$$

Hence we have $c_{m}=0,(m>0)$, and $b_{m}=0,(m<0),\left(c_{m}=0,(m \leqq 0)\right)$ considering the behavior at $\infty$ (resp. at 0 ). Thus $\Psi(w)$ is holomorphic on $H$. We show $\eta^{\lambda} \Psi(w)=0(\eta+1 / \eta)$. Using the notation in $\S 0$,

$$
\left|\theta\left(z, \sigma_{4 w}\right)\right| \leqq v^{(3-\kappa) / 4} \sum_{x \in L^{\prime}}\left|f\left(\sqrt{v} \sigma_{4 w}^{-1}\right)\right| .
$$

Put $M=Z / 4 \oplus Z / 4 \oplus Z / 4$, then

$$
\sum_{x \in L^{\prime}}\left|f\left(\sqrt{v} \sigma_{4 w}^{-1} x\right)\right| \leqq \sum_{x \in M}\left|f\left(\sqrt{v} \sigma_{w}^{-1} x\right)\right|=\sum_{x \in M}\left|f\left(\sqrt{v} \sigma_{r w}^{-1}\right)\right| \quad \text { for } \gamma \in S L(2, Z) .
$$

If $\eta>c_{1}>0$ and $|\xi|<c_{2}$, then there exist $0<h_{j}(x) \in S(R), \quad(j=1,2,3)$ such that

$$
\left|\left(\begin{array}{cc}
1 & \xi / \eta \\
0 & 1
\end{array}\right) f(x)\right| \leqq h_{1}\left(x_{1}\right) h_{2}\left(x_{2}\right) h_{3}\left(x_{3}\right)
$$

for all $x=\left(x_{1}, x_{2}, x_{3}\right) \in \boldsymbol{R}^{3}$. Thus

$$
\begin{array}{r}
\sum_{x \in M}\left|f\left(\sqrt{v} \sigma_{w}^{-1} x\right)\right|=\sum_{x \in M}\left|\left(\begin{array}{cc}
\sqrt{\eta} & 0 \\
0 & \sqrt{\eta^{-1}}
\end{array}\right)\left(\begin{array}{cc}
1 & \xi / \eta \\
0 & 1
\end{array}\right) f(\sqrt{v} x)\right| \\
\leqq\left(\sum_{x_{1}} h_{1}\left(\sqrt{v} \eta^{-1} x_{1}\right)\right)\left(\sum_{x_{2}} h_{2}\left(\sqrt{v} x_{2}\right)\right)\left(\sum_{x_{2}} h_{3}\left(\sqrt{v} \eta x_{3}\right)\right)
\end{array}
$$

where $x_{j} \in \frac{1}{4} Z$. Therefore

$$
\sum_{x \in M}\left|f\left(\sqrt{v} \sigma_{w}^{-1} x\right)\right|=0\left(\left(\sqrt{v}^{-1}+1\right)^{2}\left(\sqrt{v}^{-1} \eta+1\right)\right)
$$

for $w=\xi+i \eta,\left(|\xi|<c_{2}, \eta>c_{1}>0\right)$. Put $U=\left\{w=\xi+i \eta|| \xi \mid \leqq \frac{1}{2}, \eta>0\right.$, $|w| \geqq 1\}$. Let $c_{1}<\sqrt{3} / 2, c_{2}>\frac{1}{2}$ and choose $\gamma \in S L(2, Z)$ for $w \in H$ such that $\gamma w \in U$. Then

$$
\begin{aligned}
& \sum_{x \in L^{\prime}}\left|f\left(\sqrt{v} \sigma_{4 w}^{-1} x\right)\right| \leqq \sum_{x \in M}\left|f\left(\sqrt{v} \sigma_{r w}^{-1} x\right)\right| \\
& \left.=0(\sqrt{v}-1+1)^{3}(\operatorname{Im} \gamma w+1)\right)=0\left(\left(v^{-3 / 2}+1\right)\left(\eta+\eta^{-1}\right)\right) .
\end{aligned}
$$

Thus $\left|\theta\left(z, \sigma_{4 w}\right)\right|=0\left(v^{(3-\kappa) / 4}\left(v^{-3 / 2}+1\right)\left(\eta+\eta^{-1}\right)\right)$ for all $w \in H$ and $z \in H$, and $\eta^{2} \Psi(w)=0\left(\eta+\eta^{-1}\right)$ for all $w \in H$

We can also give a different proof of the holomorphy of $\Psi$ which is expected to be extended to the case $\kappa=3,5$ with some modification. For this, it is enough to investigate the generators of $S_{\star}\left(4 N, \bar{\chi}\left(\frac{N}{*}\right)\right)$ 
which are given by Poincaré series $G_{n}(z)=\sum_{0} \chi(\mathrm{d})\left(\frac{N}{d}\right) \overline{j(\sigma, z)^{x}}|c z+\mathrm{d}|^{-\star}$ $\times \exp (2 \pi i n \sigma z),\left(\sigma=\left(\begin{array}{ll}a & b \\ c & d\end{array}\right) \in \Gamma_{\infty} \backslash \Gamma_{0}(4 N), n=1,2, \cdots\right)$. Put

$$
\begin{aligned}
\Psi_{n}(w) & =(4 \eta)^{-\lambda} \int_{\infty} v^{\kappa / 2} \bar{\theta}\left(z, \sigma_{4 w}\right) G_{n}(z) \frac{d u d v}{v^{2}} \\
& =(4 \eta)^{-\lambda} \int_{0}^{\infty} \int_{0}^{1} v^{\kappa / 2} \bar{\theta}\left(z, \sigma_{4 w}\right) e^{2 \pi i n z} \frac{d u d v}{v^{2}}
\end{aligned}
$$

Then, we can easily evaluate this integral and have $\Psi_{n}(w)=\sum_{k=1}^{h(n)} c_{n, k} \psi_{n, k}(w)$, where $c_{n, k}$ are constants and, denoting by $X_{k},(k=1, \cdots, h(n))$, representatives of $\Gamma_{0}(2 N)$-equivalence clases of

$$
\left\{X=\left(\begin{array}{ll}
2^{-1} & \\
& 2
\end{array}\right)\left(\begin{array}{ll}
x_{1} & x_{2} / 2 \\
x_{2} / 2 & x_{3}
\end{array}\right)\left(\begin{array}{ll}
2^{-1} & \\
& 2
\end{array}\right) \mid \operatorname{det} X=-\frac{n N}{4}, x \in L^{\prime}\right\},
$$

$\psi_{n, k}(w)$ is given by

$$
\begin{gathered}
\left(c_{0} w+d_{0}\right)^{-2 \lambda} \sum_{\gamma} \chi^{\prime}(\gamma)\left(a \gamma_{k} w+b\right)^{-\lambda}\left(c \gamma_{k} w+d\right)^{-\lambda}, \\
\left(\gamma=\left(\begin{array}{ll}
a & b \\
c & d
\end{array}\right) \in \Delta_{k} \backslash \Gamma_{k}\right) .
\end{gathered}
$$

The meaning of the letters $\Delta_{k}, \Gamma_{k}, \cdots$ is as follows: $\gamma_{k}=\left(\begin{array}{ll}a_{0} & b_{0} \\ c_{0} & d_{0}\end{array}\right)$ is determined by $X_{k}= \pm \frac{\sqrt{n N}}{2} \gamma_{k}^{-1}\left(\begin{array}{ll}1 & 1\end{array}\right)^{t} \gamma_{k}^{-1}$ and $\operatorname{det} \gamma_{k}=1$. We set $\Gamma_{k}$ $=\gamma_{k} \Gamma_{0}(2 N) \gamma_{k}^{-1} . \quad \Delta_{k}$ means the stabilizer in $\Gamma_{k}$ of $\gamma_{k} X_{k}^{t} \gamma_{k}$, and $\chi^{\prime}$ is a character of $\Delta_{k} \backslash \Gamma_{k}$. Thus, we have proved the holomorphy of $\Psi_{n}$, and consequently of all $\Psi$.

Now, let $\Omega(s)$ be the Mellin transform of $\Phi(i \eta)$, the convergence of which will be shown later; then

$$
\begin{aligned}
\Omega(s) & =\int_{0}^{\infty} \Phi(i \eta) \eta^{s} \frac{d \eta}{\eta} \\
& =(-1)^{\lambda}(2 N)^{\lambda}(2 N)^{-s} \int_{0}^{\infty} \Psi\left(i \eta^{-1}\right) \eta^{s-2 \lambda} \frac{d \eta}{\eta} \\
& =(-1)^{\lambda}(2 N)^{\lambda-s} 4^{-\lambda} \int_{0}^{\infty} \eta^{s-\lambda} \int_{D} v^{\kappa / 2} \bar{\theta}\left(z, \sigma_{4 i \eta-1}\right) F(z) d_{0} z \frac{d \eta}{\eta}
\end{aligned}
$$

with $d_{0} z=d u d v / v^{2}$. From the definition of $\theta(z, g)$ and the relation 


$$
(x-i y)^{\lambda}=\sum_{\varepsilon=1}^{\lambda}\left(\begin{array}{l}
\lambda \\
\varepsilon
\end{array}\right) H_{\lambda-\varepsilon}(x) H_{\varepsilon}(y)(-i)^{s},
$$

we have a simple expression

$$
\theta\left(z, \sigma_{i_{\eta}}\right)=\left(2 \sqrt{\frac{2 \pi}{N}}\right)^{-\lambda} \sum_{\varepsilon=0}^{2}\left(\begin{array}{l}
\lambda \\
\varepsilon
\end{array}\right)(-i)^{\varepsilon} \theta_{2, \lambda-\varepsilon}(z, \eta) \theta_{1, \varepsilon}(z)
$$

for $\theta\left(z, \sigma_{i \eta}\right)$, where $\theta_{2, \lambda-\varepsilon}, \theta_{1, \varepsilon}$ are the functions defined in $\S 1$. Therefore, changing the order of integration formally for a moment, justification of which will be given later, we obtain

$$
\Omega(s)=c_{1}(s) \sum_{\varepsilon=0}^{\lambda}\left(\begin{array}{l}
\lambda \\
\varepsilon
\end{array}\right) i^{\varepsilon} \int_{D} v^{\kappa / 2} F(z) \bar{\theta}_{1, \varepsilon}(z)\left[\int_{0}^{\infty} \bar{\theta}_{2, \lambda-\varepsilon}\left(z, \eta^{-1}\right) \eta^{s-\lambda} \frac{d \eta}{\eta}\right] d_{0} z
$$

with $c_{1}(s)=(-1)^{\lambda}(2 N)^{\lambda-s} 4^{s-2 \lambda}(2 \sqrt{2 \pi / N})^{-\lambda}$. Note that we can exchange the summation and the integration as above. By virtue of the different expression of $\theta_{2,8}$ given in Example 2, the integral in the bracket becomes an Eisenstein series

$$
\begin{aligned}
& \left(\sqrt{\frac{8 \pi}{N}}\right)^{\lambda-\varepsilon+1} \sqrt{2 \pi^{-1}}(-i)^{\lambda-s} v^{-\lambda+\varepsilon}\left(\frac{1}{2}\right)\left(\frac{N}{4 \pi}\right)^{(s-s-1) / 2} \\
& \quad \times \Gamma\left(\frac{s-\varepsilon+1}{2}\right)_{x_{1} \in Z, x_{2} \in Z} \chi_{1}\left(x_{1}\right)\left(x_{1} z+x_{2}\right)^{\lambda-\varepsilon}\left|x_{1} z+x_{2}\right|^{-s+\varepsilon-1} .
\end{aligned}
$$

Changing the variable $z$ to $-1 / 4 N z$ and using $G(z)=F(-1 / 4 N z)(4 N)^{-\kappa / 4}$ $\times(-i z)^{-\kappa / 2}$ and

$$
\theta_{1, \varsigma}(-1 / 4 N z)=(2 N)^{\varepsilon / 2}(\sqrt{-2 i z})^{2 \varepsilon+1} \theta_{\varepsilon}(z)
$$

we obtain

$$
\Omega(s)=c_{2}(s) \sum_{\varepsilon=0}^{\lambda}\left(\begin{array}{l}
\lambda \\
\varepsilon
\end{array}\right)(\sqrt{2 \pi})^{\lambda-\varepsilon+1} i^{s-\lambda} I_{\varepsilon}(s)
$$

where $c_{2}(s)$ is like $c_{1}(s)$ above and $I_{\varepsilon}(s)$ is given by

$$
\begin{aligned}
I_{\varepsilon}(s)= & \int_{D} G(z) \bar{\theta}_{\varepsilon}(z) v^{(s+\varepsilon+2) / 2} \pi^{-(s-\varepsilon+1) / 2} \Gamma\left(\frac{s-\varepsilon+1}{2}\right) \\
& \times \sum_{x_{1}, x_{2} \in Z} \chi_{1}\left(x_{1}\right)\left(4 N x_{2} z+x_{1}\right)^{\lambda-\varepsilon}\left|4 N x_{2} z+x_{1}\right|^{-s+\varepsilon-1} d_{0} z \\
= & \pi^{-(s-\varepsilon+1) / 2} \Gamma\left(\frac{s-\varepsilon+1}{2}\right) L\left(s-\lambda+1, \chi_{1}\right) \\
& \times \int_{0}^{\infty} \int_{0}^{1} G(z) \bar{\theta}_{s}(z) v^{(s+\varepsilon+2) / 2} d_{0} z
\end{aligned}
$$


containing Rankin's arguments. We note that $\theta_{\varepsilon}(z)=0$ if $\varepsilon$ is odd. The convolution appearing in $I_{s}(s)$ is easily computed by the Fourier expansion $\theta_{\mathrm{s}}(z)=\sum_{k=-\infty}^{\infty}(2 v)^{-\varepsilon / 2} H_{\mathrm{c}}(2 \sqrt{2 \pi v} k) \exp \left(2 \pi k^{2} z\right)$ and by the partial integration, that is,

$$
\begin{aligned}
& \int_{0}^{\infty} \int_{0}^{1} G(z) \bar{\theta}_{\varepsilon}(z) d u v^{(s+\varepsilon) / 2} \frac{d v}{v} \\
& \quad=2^{1-\varepsilon}(4 \pi)^{-s / 2}(s-1)(s-2) \cdots(s-\varepsilon) \Gamma\left(\frac{s-\varepsilon}{2}\right) D(s),
\end{aligned}
$$

where $D(s)=\sum_{k=1}^{\infty} a\left(k^{2}\right) k^{-s}$ with $G(z)=\sum_{k=1}^{\infty} a(k) \exp (2 \pi i k z)$. Using well known formulas of $\Gamma$-function, we obtain

$$
I_{\varepsilon}(s)=2^{2-2 s} \pi^{-s+\varepsilon / 2} \Gamma(s) L\left(s-\lambda+1, \chi_{1}\right) D(s) .
$$

consequently, we have

$$
\Omega(s)=c(2 \pi)^{-s} \Gamma(s) L\left(s-\lambda+1, \chi_{1}\right) D(s),
$$

where $c$ is the constant given in $\S .0$.

There still remains the investigation of the asymptotic behavior of $\Phi(i \eta)$ as $\eta \rightarrow 0$ and $\infty$. But, it is exactly same as that in Shimura's paper. In fact, $\theta_{2, \lambda-\epsilon}(z, \eta)$ is majorized by $\eta^{-\lambda+\varepsilon-1} v^{-\lambda+\varepsilon} F_{\epsilon}(z, \eta)$, where $F_{\epsilon}(z, \eta)$ is given by

$$
\begin{aligned}
F_{\iota}(z, \eta)=\sum_{x_{1}, x_{2}}\left|x_{1} z+x_{2}\right|^{2-\varepsilon} \exp (- & \left.\frac{4 \pi}{N \eta^{2} v}\left|x_{1} z+x_{2}\right|^{2}\right), \\
& \left(x_{1} \in Z, x_{2} \in Z,\left(x_{1}, x_{2}\right) \neq(0,0)\right) .
\end{aligned}
$$

Therefore, if $\beta$ be the smallest integer $\geqq(\lambda-\varepsilon) / 2$, then

$$
F_{\varepsilon}(z, \eta) \leqq\left\{\begin{array}{l}
\ell v^{\beta+1} e^{-\pi h / v \eta^{2}}, \eta<1, v>c>0, c<\frac{\sqrt{3}}{2}, \\
\ell^{\prime} \eta^{2(\lambda-\varepsilon+1)} v^{\beta+1} e^{-\pi \eta 2 h / v}, \eta>1, \quad v>c>0, c<\frac{\sqrt{3}}{2},
\end{array}\right.
$$

where $\ell, \ell^{\prime}$ and $h$ are positive constants depending only on $\varepsilon$ and $c$. Put $U=\left\{z=u+i v \in H|| u\left|\leqq \frac{1}{2},\right| z \mid \geqq 1\right\}$, choose $\gamma_{i} \in S L(2, Z)$ such that $\bigcup_{i=1}^{k} \gamma_{i} U \supset \mathfrak{D}$ and put $T(z)=v^{k / 2} \bar{\theta}_{1, \varepsilon}(z) F(z)$, then $T\left(\gamma_{i} z\right)=0\left(g_{i}(v)\right)$ for $z \in U$ where $g_{i}$ 's are some rapidly decreasing functions. Put $F_{s}^{\prime}(z, \eta)$ $=\eta^{-\lambda+s-1} v^{-\lambda+\varepsilon} F_{\varsigma}(z, \eta)$, then $\int_{D}\left|T(z) \bar{\theta}_{2, \lambda-\varsigma}\left(z, \eta^{-1}\right)\right| d_{0} z \leqq \sum_{i=1}^{k} c_{i} \int_{U} T\left(\gamma_{i} z\right) F_{\epsilon}^{\prime}\left(\gamma_{i} z, \eta^{-1}\right)$ 
$\times d_{0} z \leqq \sum_{i=1}^{k} e_{i} \int_{c}^{\infty} v^{\nu} \eta^{\alpha} g_{i}(v) \exp \left(-\pi \eta^{2} h v^{-1}\right) d v$ hold for all $\eta>1$ with some constants $c_{i}, e_{i}, \nu_{i}, \alpha$. Since $\eta^{2 \mu} v^{-\mu} \exp \left(-\pi \eta^{2} h v^{-1}\right)<C_{\mu}$ for $\mu>0$ with some constant $C_{\mu}$ and $\eta^{2 \mu-\alpha} \int_{c}^{\infty} v^{\nu i} \eta^{\alpha} g_{i}(v) \exp \left(-\pi \eta^{2} h v^{-1}\right) d v<C_{\mu} \int_{c}^{\infty} v^{\nu_{i+\mu}} g_{i}(v) d v=C_{\mu}^{\prime}$ with some constant $C_{\mu}^{\prime}$, then $\int_{D}\left|T(z) \bar{\theta}_{2, \lambda-8}\left(z, \eta^{-1}\right)\right| d_{0} z=0\left(\eta^{-\mu}\right)$ for any $\mu>0$, $(\eta>1)$. In the same way we get $=0\left(\eta^{\mu}\right)$ for any $\mu>0, \quad(\eta<1)$. Thus, $T(z) \bar{\theta}_{2, \lambda-s}\left(z, \eta^{-1}\right) \eta^{s}$ is integrable on $(z, \eta) \in \mathfrak{D} \times R^{+}$. Hence the formal computations are justified, and the theorem is proved.

\section{§3. Supplementary discussions.}

We assume $\kappa \geq 7$. The main theorem of Shimura [1] is as follows. Let $G(z)=\sum_{n=1}^{\infty} a(n) e(n z)$ be an element of $S_{x}(4 N, \chi)$, let $t$ be a squarefree positive integer, let $\chi_{t}=\chi\left(\frac{-1}{*}\right)^{2}\left(\frac{t}{*}\right)$ be a character, and put $\Phi_{t}(w)$ $=\sum_{n=1}^{\infty} A_{t}(n) e(n w)$ with $A_{t}(n)$ determined by the equality

$$
\sum_{n=1}^{\infty} A_{t}(n) n^{-s}=\left(\sum_{m=1}^{\infty} \chi_{t}(m) m^{\lambda-1-s}\right)\left(\sum_{m=1}^{\infty} a\left(t m^{2}\right) m^{-s}\right) .
$$

Then, $\Phi_{t}$ belongs to $\widetilde{S}_{\boldsymbol{k}-1}\left(N_{t}, \chi^{2}\right)$ with a certain positive integer $N_{t}$.

In $\S 4$ of [1], he conjectured that $2 N$ can be taken as $N_{t}$.

COROLlARY TO THE THEOREM. The above conjecture is true.

Proof. Since $G(t z)=\sum_{n=1}^{\infty} b(n) e(n z)$ belongs to $S_{k}\left(4 t N, \chi\left(\frac{t}{*}\right)\right)$, our theorem implies that $\Phi^{\prime}(w)=\sum_{n=1}^{\infty} B_{1}(n) e(n w)$, determined by the equality

$$
\sum_{n=1}^{\infty} B_{1}(n) n^{-s}=\left(\sum_{m=1}^{\infty} \chi_{t}(m) m^{2-1-s}\right)\left(\sum_{m=1}^{\infty} b\left(m^{2}\right) m^{-s}\right),
$$

belongs to $\widetilde{S}_{k-1}\left(2 t N, \chi^{2}\right)$. Since $b\left(m^{2}\right)$ is equal to $a\left(t k^{2}\right)$ or 0 according as $m=t k$ or $t$ does not divide $m$,

$$
\sum_{n=1}^{\infty} B_{1}(n) n^{-s}=\left(\sum_{m=1}^{\infty} A_{t}(m) m^{-s}\right) t^{-s}
$$

holds and so $B_{1}(n)=A_{t}(n / t)$ or 0 according as $t \mid n$ or $t \times n$. Hence, $\Phi^{\prime}(w)=\Phi_{t}(t w)$, and so $\Phi_{t}(\sigma w)=(c w+d)^{2 \lambda} \chi^{2}(d) \Phi_{t}(w)$ for all $\sigma=\left(\begin{array}{ll}a & b \\ c & d\end{array}\right)$ $\in \Gamma_{0}^{t}(2 N)$ with $\Gamma_{0}^{t}(2 N)=\left\{\left(\begin{array}{ll}a & b \\ c & d\end{array}\right) \in \Gamma_{0}(2 N) \mid b \equiv 0(t)\right\}$. Put $\Gamma_{\infty}=\left\{\left(\begin{array}{ll}1 & b \\ 0 & 1\end{array}\right) \mid b\right.$ 
$\in Z\}$. Then, since $\Gamma_{0}(2 N)$ is generated by $\Gamma_{\infty}$ and $\Gamma_{0}^{t}(2 N), \Phi_{t}$ belongs to $\widetilde{S}_{k-1}\left(2 N, \chi^{2}\right)$.

By considering the reproducing kernel function of the space $S_{\star}\left(4 N, \bar{\chi}\left(\frac{N}{*}\right)\right)$ and the orthogonality relation, we can also give the inverse map like Shintani's. We denote by $\varphi^{\prime}$ the map $G \rightarrow \varphi^{\prime}(G)=\Phi_{1}$ and put $\varphi=c \varphi^{\prime}$ with the constant in Theorem. Denote by $\psi$ the map $F \rightarrow \psi(F)=\Psi$ with the notation used in the first paragraph of $\S .2$. Then, our theorem says that the diagram

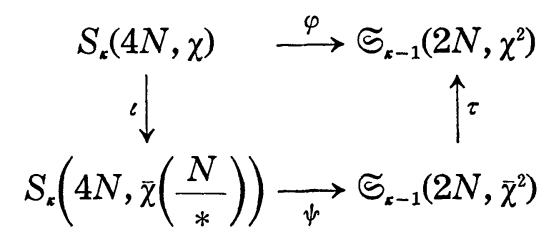

is commutative, where we denote by $\iota, \tau$ the maps $G(z) \rightarrow \iota(G)(z)$ $=G(-1 / 4 N z)(4 N)^{-\kappa / 4}(-i z)^{-\kappa / 2}, \Psi(w) \rightarrow \tau(\Psi)(w)=\Psi(-1 / 2 N w)(2 N)^{2}(-2 N w)^{-2 \lambda}$, respectively. For the sake of simplicity, we assume that $\varphi^{\prime}$ is bijective and that $G_{i},(i=1, \cdots, d)$, are the common eigen-functions of the Hecke operators which form a basis of $S_{\imath}(4 N, \chi)$. Put $F_{i}=\iota\left(G_{i}\right), \Psi_{i}=\left(\tau^{-1} \circ \varphi\right)\left(G_{i}\right)$ and $\Phi_{i}=\varphi\left(G_{i}\right)$. Then, $F_{i}, \Psi_{i}$ and $\Phi_{i}$ are the orthogonal basis of $S$ $=S_{\kappa}\left(4 N, \bar{\chi}\left(\frac{N}{*}\right)\right), \mathfrak{S}_{x-1}\left(2 N, \bar{\chi}^{2}\right)$ and $\widetilde{S}_{x-1}\left(2 N, \chi^{2}\right)$, respectively, because $\Phi_{i}{ }^{\prime} \mathrm{s}$ also are the common eigen-functions of the Hecke operators. We denote by $(*, *)$ the Petersson inner product. Put $K\left(z, z^{\prime}\right)=\sum_{i=1}^{d} c_{i} F_{i}(z) \overline{F_{i}\left(z^{\prime}\right)}$ with $c_{i}=\left(F_{i}, F_{i}\right)^{-1}$, then $K\left(z, z^{\prime}\right)$ is a reproducing kernel function of $S$, i.e. $\left(F(z), K\left(z, z^{\prime}\right)\right)=F\left(z^{\prime}\right)$. Define $\tilde{\theta}(z, w)=\left(K\left(z, z^{\prime}\right), \eta^{-2} \theta\left(z^{\prime}, \sigma_{4 w}\right)\right)$, then, we have $(F(z), \tilde{\theta}(z, w))=\left(F(z), \eta^{-2} \theta\left(z, \sigma_{4 w}\right)\right)=\psi(F)(w)$ and $\tilde{\theta}(z, w)$ $=\sum_{i=1}^{d} c_{i} F_{i}(z) \bar{\Psi}_{i}(w)$. Therefore, $\left(\tilde{\theta}(z, w), \bar{\Psi}_{i}(w)\right)=c_{i}\left(\Psi_{i}, \Psi_{i}\right) F_{i}(z) . \quad$ Put $F(z)=\left(\theta\left(z, \sigma_{4 w}\right), \bar{\Psi}_{i}(w)\right)$. Then, as is proved in [2] for a special case or can be proved with slight modification for many other cases, $F(z)$ belongs to $S$, hence $F(z)=c_{i}\left(\Psi_{i}, \Psi_{i}\right) F_{i}(z)$, and we obtain a way to get the invere image of $\varphi^{\prime}$ as far as the common eigen-functions concern.

In [3], K. Doi and H. Naganuma constructed a Hilbert modular form with respect to a real quadratic field $Q(\sqrt{D})$ from an integral modular form of one variable. This situation is similar to that of [1], and the methods of the proofs of [1], [3] are the same. Thus, [3] can 
be reformulated by means of the ideas of this paper. In this paper, we used the theta series of a ternary zero form, and the discussion was based on the classical isomorphism, $O(2,1) \approx S L(2, R)$. We can find a quaternary quadratic form $Q$ with signature $(2,2)$, which does not split over $\boldsymbol{Q}$ but splits over $Q(\sqrt{D})$, such that the orthogonal group of $Q$ is isomorphic to $S L(2, R) \times S L(2, R)$ and the group of units of $Q$ corresponds to the Hilbert modular group with respect to $Q(\sqrt{D})$. The theta series of $Q$ takes the place of $\theta(z, g)$, in this case, and the inner product of this theta series with a cusp form of one variable gives rise to a Hilbert modular form.

\section{REFERENCES}

[1] G. Shimura, On modular forms of half integral weight, Ann. of Math., 97 (1973), 440-481.

[2] T. Shintani, On construction of holomorphic cusp forms of half-integral weight, to appear.

[ 3 ] K. Doi and H. Naganuma, On the functional equation of certain Dirichlet series, Inventiones Math., 9 (1969), 1-14.

Nagoya University 